\title{
Hepatic Secretion of Lipoproteins in the Rat and the Effect of Experimental Nephrosis
}

\author{
Julian B. Marsh and Charles E. Sparks, Department of Physiology and \\ Biochemistry, The Medical College of Pennsylvania, Philadelphia, Pennsylvania \\ 1.912 .9
}

A B S T R A C T Livers from normal and nephrotic rats were perfused by the nonrecirculating technique. Nephrosis was studied on the 7th d after the injection of puromycin animonucleoside. Amino acid-labeled lipoproteins $(d<1.21)$ were isolated from the perfusion medium by agarose column chromatography or by sequential density ultracentrifugation. In both groups of animals, in addition to very low density lipoproteins and nascent high density lipoproteins, column chromatography revealed the presence of a peak of $2-3$ $\times 10^{6}$ daltons. This peak contained lipoproteins of densities corresponding to $<1.006,1.006<d<1.02$, and $1.02<d<1.06$, which indicated that rat liver secretes a heterogeneous mixture of triglyceride-rich lipoproteins.

The amount of these lipoprotein density classes was measured and their lipid and apoprotein composition and their apoprotein specific activity were determined. In both groups of rats there was a progressive rise in phospholipid and decrease in triglyceride content as the isolation density increased from 1.006 and 1.06 . The lipoproteins from the nephrotics had higher amounts of cholesterol. The livers from the nephrotic rats secreted two to three times as much lipoprotein as controls in all density classes in the first $20 \mathrm{~min}$, but during the next 40 min only the $1.02<d<1.06$ and nascent high density lipoproteins remained at this high level compared to controls. A larger total liver pool of apolipoproteins in nephrotic livers was inferred from their lower specific activities during the first $20 \mathrm{~min}$.

The apoprotein composition of liver perfusate lipoproteins from nephrotics differed from controls. There was a $40 \%$ decrease in the amount of low molecular weight apoproteins in all density classes, with corresponding increases in apo $\mathrm{B}$ and apo $\mathrm{E}$ in the tri-

A preliminary report of this work was presented to the American Society of Nephrology (1978. 11th Annual Meeting Abstracts. 108A).

Received for publication 8 February 1.979 and in revised form 2.9 June 1.97 .9 . glyceride-rich fractions. The apo A-1 content of nascent HDL was increased from $16 \%$ in controls to $52 \%$ in nephrotics, with corresponding decreases in apo $\mathrm{C}$ and apo $\mathrm{E}$. When these results were combined with specific activity measurements of the individual apoproteins and the net secretion rate of total protein in each lipoprotein class, it was possible to estimate the total amount of each apoprotein secreted and the total incorporation of labeled amino acids into each. The incorporation of label gave results similar to those obtained by direct measurement of the amounts of apoproteins. Apo $\mathrm{E}$ secretion was increased by a factor of 1.8 , apo $\mathrm{B}$ by 2.8 , and apo A-1 by 8.4 , whereas the secretion of apo $\mathrm{C}$ was not significantly altered.

We explain these results by postulating that the primary stimulus to hepatic plasma protein synthesis in response to proteinuria is general and that subsequent negative feedback regulation affects individual apolipoprotein synthesis rates. A corollary of this hypothesis is that the biosynthesis and secretion of an apoprotein may be regulated independently of the lipoprotein density class in which it is found.

\section{INTRODUCTION}

The nephrotic syndrome provides an excellent model of endogenous hyperlipemia. Marsh and Drabkin (1) and Radding and Steinberg (2) demonstrated increased hepatic synthesis of lipoproteins in experimental nephrosis produced in rats with antikidney serum. Since that time, much has been learned about the individual apoproteins, but there have been no studies of the synthesis and secretion of these apoproteins in experimental nephrosis. We have, therefore, undertaken a reinvestigation of this in rats with puromycin aminonucleoside-induced nephrotic syndrome using the single-pass liver perfusion technique developed in this laboratory (3). Four lipoprotein density classes were isolated from liver perfusates of normal and nephrotic rats, and the major apoproteins and lipids of these were measured. Incorporation of labeled amino 
acids into the lipoprotein apoproteins was measured simultaneously. The results confirm and extend previous observations concerning the heterogeneity of lipoproteins secreted by the rat liver. They explain some of the alterations of the lipoproteins in nephrotic rat plasma that have recently been determined (4). This work suggests that the synthesis of the apoproteins is independently controlled, since in nephrosis the synthesis of low molecular weight apoproteins was minimally increased while the synthesis of apo A-1 showed an eightfold increase. Clues to the role of lipoprotein catabolism in determining plasma lipoprotein concentrations and compositions can be obtained by comparison of secretory and circulatory lipoproteins, and future investigations on the feedback regulation of lipoprotein synthesis are suggested by these experiments.

\section{METHODS}

Introduction of nephrosis. The rats used in this study were male albinos of the Fisher 344 strain weighing $285 \pm 6.5 \mathrm{~g}$ and fed ad lib on Purina laboratory chow (Ralston Purina Co., St. Louis, Mo.). Nephrosis was induced by a single intraperitoneal injection of $100 \mathrm{mg} / \mathrm{kg}$ of puromycin aminonucleoside (lot 1673, ICN Nutritional Biochemicals, Cleveland, Ohio) in a $2 \%$ solution adjusted to $\mathrm{pH} 7$ with $\mathrm{HCl}$. Liver perfusions were performed between 9 and $11 \mathrm{a} . \mathrm{m}$. on the 7 th $d$ after injection. The rats showed ascites at this time and the average plasma cholesterol level was $320 \mathrm{ng} / \mathrm{dl}$, which is about four times the control level.

Liver perfusion. Rats were anesthetized by intraperitoneal injection of $30 \mathrm{mg} / \mathrm{kg}$ of nembutal (nephrotic) or $60 \mathrm{mg} / \mathrm{kg}$ (controls) and perfusion of the liver was carried out at $37^{\circ} \mathrm{C}$ by the single-pass method (3) with Krebs-Ringer bicarbonate $-0.1 \%$ glucose solution equilibrated with $95 \% \mathrm{O}_{2}-5 \% \mathrm{CO}_{2}$, $\mathrm{pH} \mathrm{7.4}$, at a flow-rate of $25-30 \mathrm{ml} / \mathrm{min}$. ${ }^{3} \mathrm{H}$-labeled L-amino acids (New England Nuclear, Boston, Mass., NET 250, average sp act, $29 \mathrm{Ci} / \mathrm{mm}$ ) were present at a concentration of $0.1 \mathrm{Ci} / \mathrm{ml}$. After an initial 3-min perfusion to remove blood, the perfusate was collected.

Lipoprotein isolation. All operations were carried out at $4^{\circ} \mathrm{C}$. Perfusates were concentrated in a TCF-10 apparatus using $90 \mathrm{~cm}$ PM-10 ultrafiltration membranes (Amicon Corp., Scientific Sys. Div., Lexington, Mass.). Control experiments indicated that $86-90 \%$ of the radioactivity of ${ }^{125}$ I-labeled very low density lipoproteins (VLDL) ${ }^{1}$ was recovered. Lipoprotein isolation was carried out by sequential density flotation in the Beckman L3-50 ultracentrifuge using the Beckman Ti50 rotor (Beckman Instruments, Inc., Spinco Div., Palo Alto, Calif.) at salt densities of $1.006,1.02,1.06$, and 1.21 using $\mathrm{NaBr}$ for density adjustment (3). The speeds and times were $45,000 \mathrm{rpm}(134,000 \mathrm{~g})$ and $18-24 \mathrm{~h}$ for the $d<1.06$ fractions and 50,000 rpm $(165,000 \mathrm{~g})$ and $48 \mathrm{~h}$ for high density lipoproteins (HDL). After centrifugation, the top $10 \%$ of the volume of each tube was removed except in the case of HDL, where the top 5\% was removed. The HDL results were corrected for $85 \%$ recovery in the top $5 \%$, compared to the top $10 \%$ as determined in control experiments with ${ }^{125}$ I-labeled HDL. All isolated lipoproteins were dialyzed overnight against

\footnotetext{
${ }^{1}$ Abbreviations used in this paper: HDL, high density lipoproteins $(1.06<d<1.21)$; PAN, puromycin aminonucleoside ( $3^{\prime}$-amino- $N^{6}$-dimethyl adenosine); SDS, sodium dodecyl sulfate; VLDL, very low density lipoproteins $(d<1.006)$.
}

at least $200 \mathrm{vol}$ of $0.15 \mathrm{M} \mathrm{NaCl}-2 \mathrm{mM}$ EDTA, pH 7.4, using dialysis membranes having a 3,500-dalton exclusion (Arthur H. Thomas Co., Philadelphia, Pa.).

In some experiments, total lipoproteins were isolated at $d<1.21$ and analyzed by column chromatography on agarose A-15 M (Bio-Rad Laboratories, Richmond, Calif.) by the method of Rudel et al. (5).

Lipoprotein analysis. After isolation, $1 \mathrm{mg}$ of dextran T-500 (Pharmacia Fine Chemicals Co., Piscataway, N. J.) was added to each fraction and the solution was concentrated to $0.5 \mathrm{ml}$ using CF-25 centricones (Amicon Co.). Less than 5\% of the Lowry-reactive protein was present in the ultrafiltrate. Delipidation was then carried out by the method of Lux et al. (6), which employs $20 \mathrm{vol}$ of $2: 1(\mathrm{vol} / \mathrm{vol})$ chloroform-methanol and $20 \mathrm{vol}$ of ether, followed by washing of the precipitate with ether. The combined solvent extracts were taken to dryness at $50^{\circ} \mathrm{C}$ under reduced pressure in a flash evaporator, and redissolved in 2:1 chloroform-methanol. The extract was treated with $0.2 \mathrm{vol}$ of $0.15 \mathrm{M} \mathrm{NaCl}$ and the chloroform phase was used for thin-layer chromatographic separation and measurement of lipid classes by the sulfuric acid charring method (7). Cholesterol and cholesterol esters were measured directly by the addition of acetic acid, ferric perchlorate, and sulfuric acid to the scraped silica gel, which was removed by centrifugation before absorbancy measurements (8).

The apoprotein-dextran precipitate after delipidation was dissolved in $1 \%$ sodium dodecyl sulfate (SDS), $1 \% 2$-mercaptoethanol, heated for $2 \mathrm{~min}$ at $100^{\circ} \mathrm{C}$, and the apoproteins were separated in $0.1 \%$ SDS in $10 \%$ polyacrylamide gels. Staining with Coomassie Blue and densitometry were carried out as described (9). The results were corrected for differential dye binding and for losses during the staining procedure according to the general method of Kane et al. (10) by means of standard curves constructed with known amounts of apoproteins isolated from plasma lipoproteins by column chromatography on Sepharose CL-6B (Pharmacia Fine Chemicals) in the presence of $1 \%$ SDS (9). Apo B, apo E, and the apo C mixture were prepared from plasma VLDL and apo A-1 from plasma HDL. Apo B was assigned a correction factor of 1.0 and, on a comparative basis, apo $\mathrm{E}$ and apo $\mathrm{A}-1$ averaged 1.7 and apo $\mathrm{C}$, 2.1. These correction values include solubility losses during staining and are not measurements of dye binding per se. The individual apoproteins were identified on the basis of electrophoretic mobility in SDS gels, with standards, as described $(9,11)$. Apo B was measured as the sum of two distinct high molecular weight bands that just entered the gels; these bands were absent when apoprotein precipitates were extracted with $50 \%$ tetramethyl urea in which apo B is not soluble. The designation apo $\mathrm{C}$ is given to the entire group of apoproteins (apo C-I, C-II, C-III, and A-II) which have molecular weights less than A-1.

Protein concentrations were determined by the Lowry (12) method using bovine serum albumin as a standard and adding $1 \%$ SDS when necessary to prevent interference from turbidity. Radioactivity measurements were made by liquid scintillation spectrometry, using an internal standard for quench corrections and the counting error was $\pm 5 \%$. Specific activities of individual apoproteins were measured by scanning densitometry to determine the relative amounts of each apoprotein, followed by slicing each gel and treatment of each slice with $30 \% \mathrm{H}_{2} \mathrm{O}_{2}$ at $56^{\circ} \mathrm{C}$ for $8-12 \mathrm{~h}$ before addition of the scintillation fluid. Recoveries of ${ }^{3} \mathrm{H}$-apoproteins av. ged $86 \pm 1.5 \%$ (SD) in three experiments.

\section{RESULTS}

The size distribution of amino acid-labeled lipoproteins secreted by normal and nephrotic rat liver is shown in 
Fig. 1. The first peak, corresponding to the void volume of the column, floated at $d<1.006$ and its lipid and apoprotein composition was that of VLDL. The second peak, with an $\cong 2-3 \times 10^{6} \mathrm{~mol}$ wt, was heterogeneous with regard to density; about one third of the total floated at each successive density of 1.006, 1.02, and 1.06. The third peak, corresponding to an $\cong 5 \times 10^{5}$ mol wt, contained apo A-1 and apo $\mathrm{E}$ and its lipid composition corresponded to that of nascent HDL isolated from liver perfusates by sequential density ultracentrifugation (9). A control perfusion was carried out in the absence of labeled amino acids and the $d<1.21$ fraction was acetylated in vitro with ${ }^{3} \mathrm{H}$-acetic anhydride (13). As shown in Fig. 2, a similar distribution of labeled lipoproteins was found after column chromatography, indicating that the amino acid-labeled lipoproteins were representative of the total mass of perfusate lipoproteins.

When liver perfusates from control and nephrotic rats were subjected to sequential density ultracentrifugation, four density fractions were obtained. During the first 20 min of perfusion, there were two to threefold increases in the secretion of all four lipoprotein density classes, as well as total protein output, in the perfusates of livers from the nephrotic rats (Table I). During the next $40 \mathrm{~min}$, the rate of protein and of lipoprotein

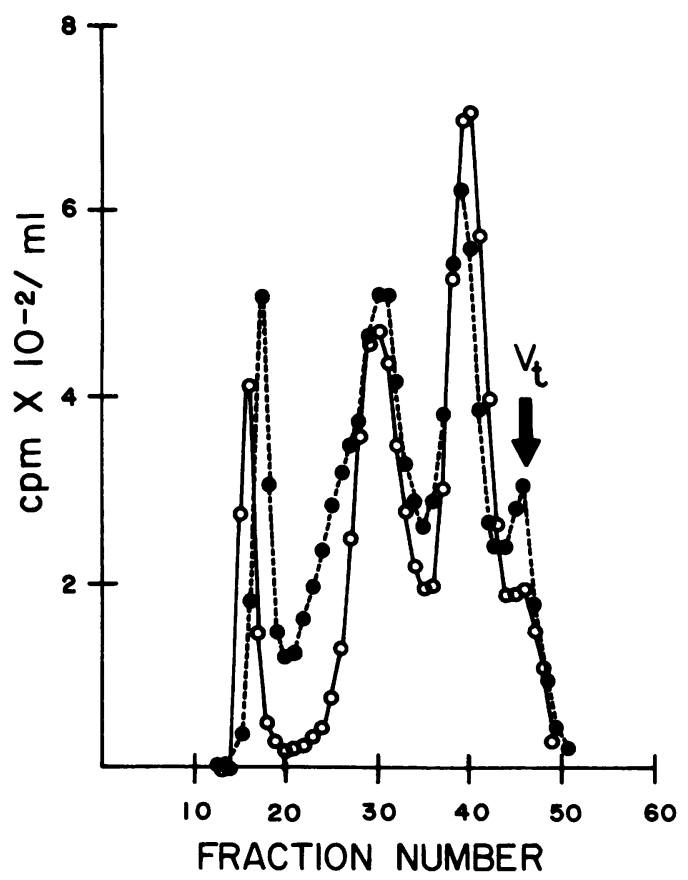

FIGURE 1 Agarose column chromatography of ${ }^{3} \mathrm{H}$-amino-acidlabeled $d<1.21$ perfusate lipoproteins. An approximately equal amount of labeled lipoprotein, $d<1.21$, from each perfusate was applied to a $1.6 \times 90-\mathrm{cm}$ column of agarose $\mathrm{A}-15 \mathrm{M}$ and 4-ml fractions were collected. (๑) Control liver perfusate; (O), nephrotic liver perfusate.

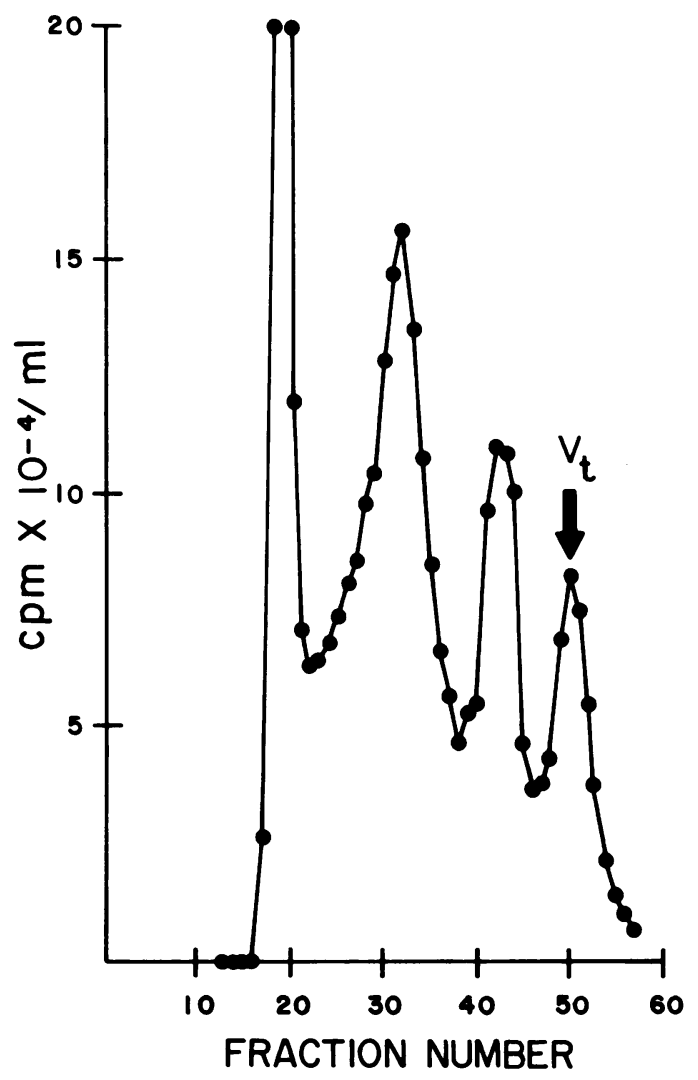

FIGURE 2 Agarose column chromatography of control liver perfusate $d<1.21$ lipoproteins labeled in vitro with ${ }^{3} \mathrm{H}$-acetic anhydride. The labeled lipoproteins, after dialysis, were applied to a $1.6 \times 90-\mathrm{cm}$ column of agarose A-15M and $3.6-\mathrm{ml}$ fractions collected.

release declined by about one-third but the livers of the nephrotic animals still evidenced significant one to twofold increases in total protein, and in the $1.006<d$ $<1.02$ and $1.06 d<1.21$ (nascent HDL) fraction outputs. The most striking change, in comparison with the initial perfusion period, was the considerable decline $(61 \%)$ in VLDL output in the nephrotic rats, while the controls continued to release VLDL at $82 \%$ of the initial rate.

The lipid and apoprotein composition of the perfusate lipoproteins is shown in Tables II and III. Analysis of the data from the two perfusion periods indicated no significant differences and the analyses were combined in Tables II and III. The progressive decline in triglyceride content of the $d<1.06$ fractions is evident in both groups of rats (Table II). Significant increases in the cholesterol content of these lipoprotein fractions were found in the nephrotic group. The apoprotein composition (Table III) showed differences between nephrotics and controls in every lipoprotein fraction. As with the lipid composition, there were no significant differences between the first 20 and the second $40 \mathrm{~min}$ 
TABLE I

Lipoprotein Secretion by Perfused Liver

\begin{tabular}{|c|c|c|c|c|c|c|}
\hline \multirow[b]{3}{*}{ Lipoprotein class } & \multicolumn{6}{|c|}{ Perfusion time } \\
\hline & \multicolumn{3}{|c|}{20} & \multicolumn{3}{|c|}{40} \\
\hline & $\mathrm{C}^{*}$ & $\mathrm{~N}^{*}$ & $\mathrm{~N} / \mathrm{C}$ & $\mathrm{C}$ & $\mathbf{N}$ & $\mathrm{N} / \mathrm{C}$ \\
\hline & \multicolumn{6}{|c|}{$\min$} \\
\hline Total protein, $m g / g / h$ & $4.44 \pm 0.49$ & $8.10 \pm 0.92 \ddagger$ & 1.8 & $2.81 \pm 0.38$ & $4.93 \pm 0.34 \ddagger$ & 1.8 \\
\hline $\begin{array}{l}\text { VLDL, } \mu g \text { protein/g/h } \\
1.006<d<1.02,\end{array}$ & $55 \pm 5.44$ & $119 \pm 12.2 \ddagger$ & 2.2 & $45 \pm 4.79$ & $47 \pm 4.87$ & 1.0 \\
\hline $\begin{array}{c}\mu g \text { protein/g/h } \\
1.02<d<1.06\end{array}$ & $33 \pm 4.92$ & $94 \pm 4.36$ & 2.8 & $22 \pm 4.17$ & $63 \pm 11.6 \ddagger$ & 2.9 \\
\hline ug protein/g/h & $21 \pm 3.48$ & $39 \pm 2.18$ & 1.9 & $13 \pm 2.81$ & $23 \pm 2.42$ & 1.8 \\
\hline HDL, $\mu g$ protein/g/h & $30 \pm 3.91$ & $87 \pm 1.77 \ddagger$ & 2.9 & $16 \pm 2.11$ & $47 \pm 8.23 \ddagger$ & 2.9 \\
\hline
\end{tabular}

* C, control; N, nephrotic.

I Significantly different from the mean of the controls $(P<0.05)$; there were five control and four nephrotic animals.

of perfusion, and the data were combined. Comparing nephrotics to controls, all lipoprotein density classes showed decreases in the $\mathrm{C}$ apoproteins. In the triglyceride-rich lipoproteins, there was a clear trend toward higher contents of apo $\mathrm{B}$ and apo $\mathrm{E}$, which was statistically significant for the $1.006<d<1.02$ fraction. Nascent HDL showed the most pronounced changes. There was a complete reversal of the normal high apo E:apo A-1 ratio in addition to the decrease in apo C.

The specific activities of the individual apoproteins were measured and the results are given in Table IV. There were no significant differences in the specific activity of a given apoprotein between lipoprotein classes in the triglyceride-rich lipoproteins; these could therefore be combined into a $d<1.06$ fraction with which the apoproteins of nascent HDL could be compared. In both controls and nephrotics, the apo B found in the nascent HDL fraction had a higher specific activity than in the $d<1.06$ fraction at the 20-min period; in the nephrotics the apo $C$ of nascent HDL had a lower specific activity after 20 and $60 \mathrm{~min}$ of perfusion. The increase in specific activity of all apoproteins after $60 \mathrm{~min}$ is evident.

Comparing nephrotics to controls, at $20 \mathrm{~min}$, all of the apoproteins had lower specific activities. After 60 min, this observation no longer held; significantly lower specific activities were only found for apo $E$ and apo $C$. In the case of apo A-1, found almost entirely in HDL, the specific activity increased $(20-60 \mathrm{~min})$ from 36 to 102 in the controls and from 9 to 63 in the nephrotics, a higher rate of increase.

The specific activity data of Table IV, the composi-

TABLE II

Lipid Composition of Perfusate Lipoproteins

\begin{tabular}{|c|c|c|c|c|c|}
\hline Lipoprotein class & $\begin{array}{l}\text { Lipid:protein } \\
\text { ratio }\end{array}$ & Phospholipids & Triglyceride & Cholesterol & $\begin{array}{l}\text { Cholesteryl } \\
\text { esters }\end{array}$ \\
\hline & & \multicolumn{4}{|c|}{$\%$ of total lipid } \\
\hline \multicolumn{6}{|l|}{ Control } \\
\hline VLDL $(12)^{*}$ & 6.9 & $40 \pm 1.71$ & $55 \pm 1.41$ & $1.9 \pm 0.42$ & $0.9 \pm 0.23$ \\
\hline $1.006<d<1.02(10)$ & 5.1 & $44 \pm 3.87$ & $48 \pm 3.94$ & $2.2 \pm 0.58$ & $1.8 \pm 0.53$ \\
\hline $1.02<d<1.06(14)$ & 3.8 & $55 \pm 2.42$ & $35 \pm 1.97$ & $1.8 \pm 0.42$ & $0.5 \pm 0.21$ \\
\hline HDL (4) & 1.3 & $66 \pm 1.50$ & $20 \pm 10.1$ & $4.6 \pm 1.78$ & $4.1 \pm 1.53$ \\
\hline \multicolumn{6}{|l|}{ Nephrotic } \\
\hline VLDL (12) & 6.8 & $42 \pm 3.87$ & $47 \pm 4.71$ & $3.8 \pm 0.50^{*}$ & $1.1 \pm 0.25$ \\
\hline $1.006<d<1.02(13)$ & 5.8 & $47 \pm 4.36$ & $41 \pm 3.88$ & $4.2 \pm 0.46^{*}$ & $2.1 \pm 0.39$ \\
\hline $1.02<d<1.06$ & 4.2 & $50 \pm 3.16$ & $38 \pm 3.70$ & $5.3 \pm 0.56^{*}$ & $3.7 \pm 0.87^{*}$ \\
\hline HDL (4) & 1.4 & $63 \pm 3.49$ & $18 \pm 5.40$ & $4.3 \pm 0.61$ & $9.0 \pm 1.29$ \\
\hline
\end{tabular}

* Significantly different from the mean of controls, $P<0.05$. The numbers in parentheses represent the number of observations. 
TABLE III

Apoprotein Composition of Perfusate Lipoproteins

\begin{tabular}{|c|c|c|c|c|}
\hline \multirow[b]{2}{*}{ Lipoprotein class } & \multicolumn{4}{|c|}{ Apoprotein } \\
\hline & B & E & $\mathrm{C}$ & $A-1$ \\
\hline & \multicolumn{4}{|c|}{$\%$ of total apoprotein } \\
\hline \multicolumn{5}{|l|}{ Control } \\
\hline VLDL $(10)^{*}$ & $17.9 \pm 1.19$ & $33.1 \pm 1.54$ & $49.1 \pm 3.43$ & \\
\hline $1.006<d<1.02(10)$ & $23.5 \pm 1.27$ & $33.7 \pm 1.32$ & $33.9 \pm 0.78$ & \\
\hline $1.02<d<1.06(8)$ & $28.2 \pm 2.62$ & $37.3 \pm 1.58$ & $25.8 \pm 1.79$ & \\
\hline HDL (10) & $6.1 \pm 0.74$ & $50.4 \pm 1.67$ & $22.0 \pm 1.71$ & $15.7 \pm 0.88$ \\
\hline \multicolumn{5}{|l|}{ Nephrotic } \\
\hline VLDL (8) & $22.6 \pm 2.22$ & $38.8 \pm 1.78 \ddagger$ & $36.1 \pm 2.86 \ddagger$ & \\
\hline $1.006<d<1.02(8)$ & $30.6 \pm 2.09$ & $46.6 \pm 2.70 \S$ & $18.1 \pm 1.36 \oint$ & \\
\hline $1.02<d<1.06(8)$ & $37.0 \pm 3.06$ & $42.4 \pm 2.12$ & $12.5 \pm 1.94 \S$ & \\
\hline HDL (8) & $4.6 \pm 1.03$ & $25.3 \pm 1.49 \S$ & $14.0 \pm 0.85 \S$ & $52.3 \pm 1.49 \S$ \\
\hline
\end{tabular}

* The numbers in parentheses represent the number of observations.

$\$$ Significantly different from mean of controls $(P<0.05)$.

$\S$ Significantly different from mean of controls $(P<0.01)$.

tion data of Table III, and the net secretion values of Table I have been combined graphically in Figs. 3A and B. Fig. 3A shows that the net secretion rate of all of the apoproteins except apo $\mathrm{C}$ was increased in the livers of nephrotic rats; the increase for apo A-1 (770\%) is especially evident compared to the low control rate. Fig. 3B shows that the net release of labeled apoproteins was also increased for all apoproteins except apo C; the increase was small (38\%) for apo $\mathrm{E}$ and large for apo B (200\%) and apo A-1 (441\%).

\section{DISCUSSION}

The single-pass liver perfusion technique employed in these studies avoids further contact of lipoproteins with the liver after initial secretion, thereby minimiz-

TABLE IV

Specific Activity of Apolipoproteins

\begin{tabular}{|c|c|c|c|c|c|c|c|c|}
\hline \multirow[b]{3}{*}{ Lipoprotein class } & \multicolumn{8}{|c|}{ Apoprotein } \\
\hline & \multicolumn{2}{|r|}{ B } & \multicolumn{2}{|r|}{$\mathrm{E}$} & \multicolumn{2}{|c|}{$\mathrm{C}$} & \multicolumn{2}{|c|}{ A-1 } \\
\hline & $\mathrm{C}^{*}$ & $N^{*}$ & C & $\mathrm{N}$ & $\mathrm{C}$ & $\mathrm{N}$ & $\mathrm{C}$ & $\mathrm{N}$ \\
\hline & \multicolumn{8}{|c|}{$\mathrm{cpm} / \mu \mathrm{g}$} \\
\hline \multicolumn{9}{|l|}{ 20-min perfusion } \\
\hline VLDL & $18 \pm 3.37$ & $8 \pm 2.20 \ddagger$ & $40 \pm 5.50$ & $14 \pm 2.66 \ddagger$ & $49 \pm 10.0$ & $15 \pm 1.58$ & & \\
\hline $1.006<d<1.02$ & $17 \pm 2.27$ & $13 \pm 0.65$ & $46 \pm 8.13$ & $15 \pm 2.48 \ddagger$ & $58 \pm 2.68$ & $43 \pm 12.1$ & & \\
\hline $1.02<d<1.06$ & $17 \pm 1.04$ & $12 \pm 1.78$ & $53 \pm 4.04$ & $19 \pm 4.33 \ddagger$ & $71 \pm 8.06$ & $52 \pm 15.2$ & & \\
\hline$d<1.06$ & $17 \pm 1.30$ & $11 \pm 1.06 \ddagger$ & $47 \pm 3.45$ & $16 \pm 1.83$ & $59 \pm 5.37$ & $37 \pm 7.61 \ddagger$ & & \\
\hline HDL & $52 \pm 12.2$ & $39 \pm 7.76$ & $25 \pm 2.18$ & $18 \pm 4.88$ & $61 \pm 10.4$ & $21 \pm 5.63$ & $36 \pm 5.00$ & $9 \pm 2.53 \$$ \\
\hline \multicolumn{9}{|l|}{ 40-min perfusion } \\
\hline VLDL & $105 \pm 20.4$ & $159 \pm 26.4$ & $197 \pm 16.2$ & $120 \pm 28.3$ & $220 \pm 46.0$ & $159 \pm 12.4$ & & \\
\hline $1.006<d<1.02$ & $95 \pm 16.2$ & $132 \pm 30.4$ & $171 \pm 16.7$ & $116 \pm 24.5$ & $173 \pm 8.46$ & $168 \pm 25.5$ & & \\
\hline $1.02<d<1.06$ & $62 \pm 10.4$ & $91 \pm 9.94$ & $146 \pm 8.2$ & $109 \pm 21.3$ & $204 \pm 28.2$ & $174 \pm 26.1$ & & \\
\hline$d<1.06$ & $86 \pm 10.5$ & $127 \pm 15.0 \ddagger$ & $174 \pm 9.52$ & $115 \pm 13.1 \ddagger$ & $201 \pm 18.7$ & $167 \pm 11.8$ & & \\
\hline HDL & $114 \pm 25.5$ & $93 \pm 20.6$ & $146 \pm 14.8$ & $116 \pm 32.0$ & $182 \pm 17.1$ & $86 \pm 20.5 \ddagger$ & $102 \pm 10.4$ & $63 \pm 14.8$ \\
\hline
\end{tabular}

* $\mathrm{C}$ and $\mathrm{N}$ represent control and nephrotic rats, respectively. All specific activities were normalized to a specific activity of $10 \mathrm{cpm} / \mu \mathrm{g}$ of liver protein.

$\ddagger$ Significantly different from the corresponding mean of the controls $(P<0.05)$. 

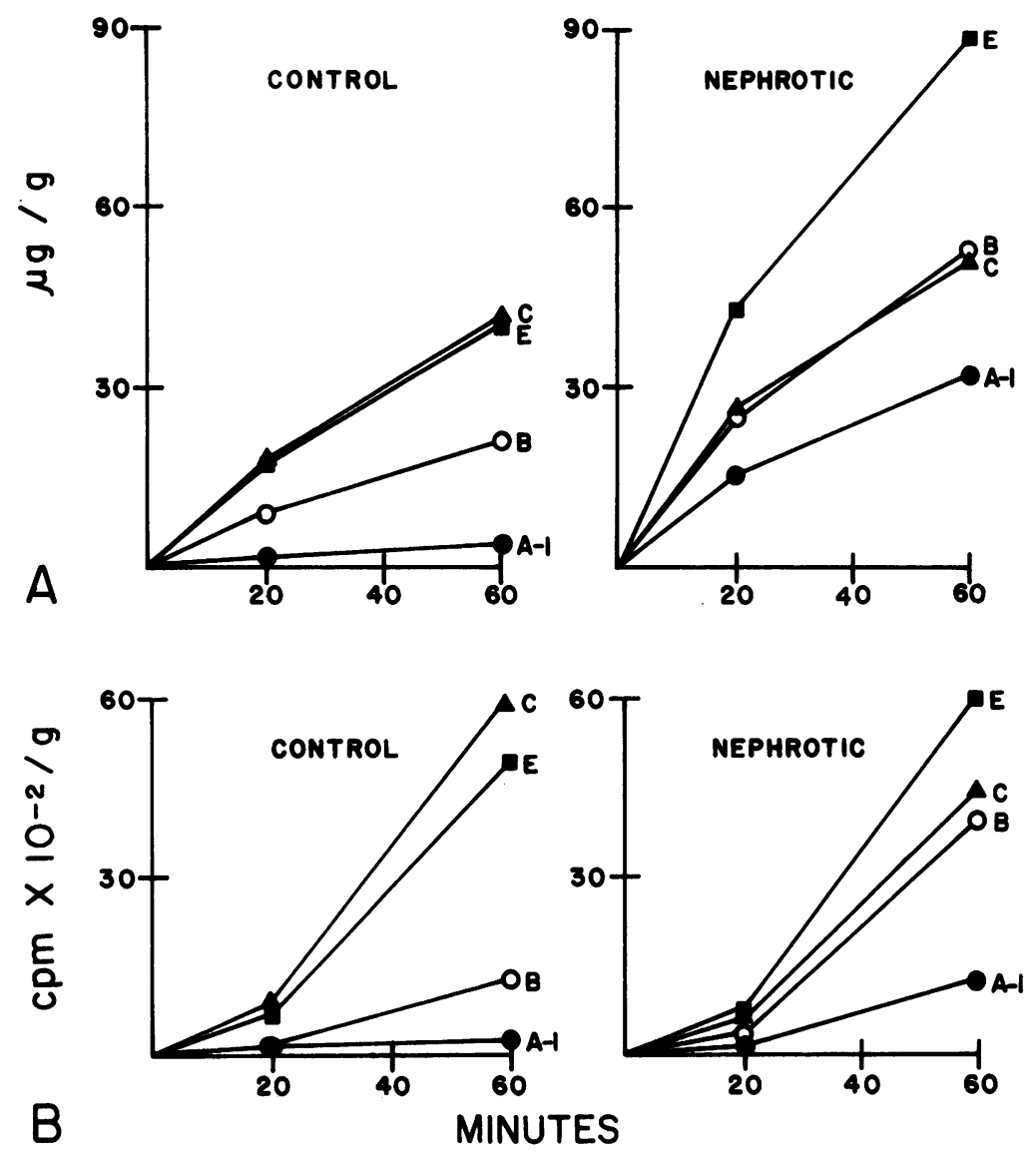

FIGURE 3 (A) Net release of apolipoproteins by perfused control and nephrotic liver. (B) Net release of labeled apolipoproteins by perfused control and nephrotic liver. $O$, apo B; 9 , apo A-1; $\Delta$, apo $\mathrm{C} ; \boldsymbol{\square}$, apo E.

ing catabolic alterations. The use of short perfusion times makes it likely that the secretory rates approach the in vivo rates at the time of the experiment. The increases in total protein and total lipoprotein output previously demonstrated in antikidney serum nephrosis (1) were found in this study with puromycin aminonucleoside (PAN) nephrosis; our previous observations were limited to apo B-rich lipoproteins but this work extends these to nascent HDL. Therefore, the increased hepatic synthesis of all classes of lipoproteins in experimental nephrosis may be regarded as firmly established. These increases occur in spite of the decreased food consumption and liver glycogen (14) of PAN nephrotic rats.

In our earlier experiments on lipoprotein secretion by the perfused normal rat liver, we found that measurable amounts of lipoproteins were recovered from the perfusate in the 1.006- to 1.06- $d$ range in addition to VLDL and nascent HDL. This lipoprotein fraction was not distinguished from VLDL except by its greater density and lower lipid content and we referred to it as a mini-VLDL. These experiments make it clear that rat liver secretes a heterogeneous spectrum of triglycer- ide-rich lipoproteins in the $d<1.06$ range. With increasing lipoprotein density, the percentage of phospholipid tends to increase, whereas that of triglyceride declines. At the same time the percentage of apo B rises, whereas that of low molecular weight apoproteins, mainly apo C's, declines, with apo E remaining about the same (Tables II and III). This density spectrum is not a continuous one with regard to particle size; separation of the entire $d<1.21$ lipoprotein fraction by column chromatography revealed a prominent peak $\cong 2-3 \times 10^{6}$ daltons, in addition to VLDL and nascent HDL. Within this peak, lipoproteins of densities, ranging from $<1.006$ to 1.06 were found. In livers of nephrotic rats secreting twice as much lipoprotein, slight changes were found in the size distribution of the labeled lipoproteins (Fig. 1). There was less lipoprotein in the area between the first and second peaks of nephrotic rat livers. The work of Nakaya et al. (15) indicated that pig liver secretes a lipoprotein resembling pig plasma LDL; in the rat a heterogeneous group of molecules in the 1.02- to 1.06- $d$ range is secreted.

The nephrotic syndrome is accompanied by substan- 
tial increases in the rate of hepatic lipoprotein synthesis and secretion. We have investigated the question of whether this increase equally involves all classes of lipoproteins and their individual apoproteins. The mechanism for the stimulation of plasma protein synthesis, including lipoproteins, in nephrosis has not been identified. Our previous studies indicated increases in liver RNA and DNA (16). All classes of lipoproteins are elevated in the plasma of nephrotic rats and as we would expect there is increased synthesis and secretion of all lipoprotein classes. In the first $20 \mathrm{~min}$ of perfusion, VLDL, the $1.006<d<1.02$ and $1.02<d<1.06$ fractions, and nascent HDL protein secretion were increased by factors of $2.2,2.8,1.9$, and 2.9 , respectively (Table I) whereas in plasma, the levels were increased by factors of $8,4,5$, and 5 (17). The increased protein secretion persisted for the next $40 \mathrm{~min}$ in all fractions except VLDL which we interpret as the release of large amounts of VLDL stored within liver Golgi vesicles of nephrotic rats in the initial 20 min of perfusion. ${ }^{2}$ The non-VLDL fractions may be continuously secreted without long periods of storage in the Golgi. The perfusion medium we have employed contains no free fatty acids; the lack of an exogenous supply of these may be rate-limiting for VLDL synthesis during the subsequent $40 \mathrm{~min}$ perfusion period.

The lipid composition of control and nephrotic secretory lipoproteins of Fisher 344 rats was not much different from that reported (3) in normal rats of the Holtzman strain. The $d<1.06$ lipoproteins of nephrotics showed increased amounts of cholesterol and cholesterol esters which may be related to the increased hepatic cholesterol content (18). The high triglyceride content of the nascent HDL that we reported earlier (3) has been confirmed. It may be related to the presence of apo $B$ in this fraction that has also been found by Hamilton et al. (19). Those investigators have shown that the apo B-containing portion of nascent HDL can be separated from the rest of this fraction by concanavalin-A affinity chromatography (20). This apo B clearly is present in a separate (probably smaller) pool, distinct from the triglyceride-rich fraction pool, because it had a significantly higher specific activity at $20 \mathrm{~min}$, in both control and nephrotic rats (Table IV).

There was minimal contamination of secreted lipoproteins by plasma lipoproteins contained in residual blood after the 3-min initial perfusion. Measurements of hemoglobin present in the perfusates indicated

${ }^{2}$ Electron microscopic study (Smith, J., J. B. Marsh, and C. E. Sparks, unpublished experiments) supports this conclusion. Before perfusion, nephrotic livers contain an enhanced endoplasmic reticulum and many Golgi membranes filled with VLDL, whereas these appear empty after perfusion for $30 \mathrm{~min}$. It is also possible that some lipoproteins, including VLDL, are bound to the liver surface and slowly released during the course of perfusion.
$<15 \mu$ l of whole blood in the initial 20 -min perfusates. The secreted HDL was quantitatively and qualitatively the same in the first 20 and the next $40 \mathrm{~min}$ of perfusion. The HDL cholesterol ester in the first $20 \mathrm{~min}$ averaged $48.7 \pm 4.5 \%$ of the total cholesterol in controls and $45.4 \pm 4.2 \%$ in nephrotics, values that are significantly less than plasma HDL, which contain about $80 \%$ cholesterol in ester form.

The apoprotein composition of perfusate lipoproteins of nephrotic rats was quite different from that of the controls, despite similarities in their lipid composition. Every lipoprotein density class in the nephrotic group had decreased amounts of low molecular weight apo-C's averaging $40 \%$. The greatest decreases were found in the $1.006<d<1.02$ and $1.02<d<1.06$ fractions $(50 \%)$. The $1.006<d<1.02$ and the nascent HDL fractions, which showed the largest increases in the nephrotics, during the second $40 \mathrm{~min}$ of perfusion, were the most profoundly altered in apoprotein composition, the former showing increases in apo $\mathrm{B}$ and apo $\mathrm{E}$, along with the low apo C. Nascent HDL of nephrotics contained so much apo A-1 that all other apoproteins were greatly reduced.

The amino acid incorporation studies suggest that these changes in apoprotein composition reflect changes in synthesis rates, as well as possible changes in intracellular or surface-bound pool sizes. We have normalized our specific activity data for the nephrotics to the same liver total protein specific activity, to minimize differences caused by the amino acid pool size. Previous studies have shown the liver free amino acid content of nephrotic livers was either unchanged (21) or decreased (22). Therefore, the decreased specific activity of the apoproteins in perfusates of nephrotics during the first $20 \mathrm{~min}$ (Table IV) coupled with the increase in the absolute amounts measured, is best interpreted as an increase in the total liver pool size. The normal 10-15 min lag in the appearance of labeled protein from the liver, which represents the time required for newly synthesized proteins released from ribosomes to traverse the secretory apparatus (23), was present in both groups of animals. The rise in total apoprotein labeling from 20 to $60 \mathrm{~min}$, shown in Fig. 3, was more pronounced in apoproteins of nephrotics than controls for all apoproteins except apo-C. Fig. 3 also shows the absolute amounts of each apoprotein released into the perfusion medium by the liver. Apo $\mathrm{C}$ release was increased only during the first $20 \mathrm{~min}$, whereas all of the other apoproteins were increased at 20 and $60 \mathrm{~min}$. For both total secretion and total labeling the degree of increase seen in perfusates of nephrotics was different for each apoprotein. The ratio of apoprotein output of nephrotics to controls (averaging both net release and total labeling; Fig. 3) for apo $\mathrm{C}$ was 1.0; for apo $\mathrm{E}$, 1.8; for apo B, 2.8; and for apo A-1, 8.4. It is possible that additional apolipoproteins may be secreted without 
sufficient lipid to appear at $d<1.21$ and apoprotein may dissociate during ultracentrifugal isolation (24), which would result in underestimates of apoprotein secretion. The values we have obtained for apo A-1 and apo $\mathrm{E}$ secretion are in excellent agreement with those estimated by Felker et al. (25) (5-12 and $40 \mu \mathrm{g} / \mathrm{g}$ per h, respectively) in recirculating perfusions in which radioimmunoassay techniques were employed for apoprotein estimation.

On the basis of this study, we can say that though the synthesis and secretion of all of the lipoprotein classes are increased in PAN nephrosis in rats, the individual apoproteins are not affected to the same extent. Our hypothesis to explain these results makes the assumption that the stimulus, still unknown, to plasma protein production in the nephrotic syndrome is general and affects all plasma proteins of hepatic origin (18). Individual differences in synthetic rate of apoproteins can be explained by postulating independent control for the synthesis or secretion of each apoprotein. In the case of apo $\mathrm{C}$, negative feedback may be very effective because of the rapid C-protein exchange between lipoprotein classes (26).

The ready exchangeability of apo $\mathrm{C}$ could account for a larger total liver pool of apo $\mathrm{C}$ in nephrotic liver especially because the plasma levels of these lipoproteins are so high. The fact that net apo $\mathrm{C}$ output was increased in the first $20 \mathrm{~min}$ of perfusion is compatible with this interpretation. It is also possible that surfacebound apo C's could be released during this time period, or that apo $\mathrm{C}$ synthesis was increased earlier in the development of the neprhrotic syndrome. After PAN administration, proteinuria begins at days 4 and 5 (27), so that at day 7 , we are approaching a steady-state plasma level at which negative feedback can occur. High plasma total apo C levels could be caused by normal synthesis rates coupled with decreased catabolism. There is evidence for decreased catabolism of plasma proteins in nephrosis $(28,29)$. Further work is needed to substantiate our hypothesis and to explore the details of the regulation of apoprotein synthesis by the liver. Experimental nephrosis in rats is a useful means of investigating normal regulatory mechanisms of lipoprotein and other plasma protein synthesis and secretion.

\section{ACKNOWLEDGMENTS}

We are indebted to Ms. Genevieve Stoudt and Dr. George Rothblat for the nascent HDL cholesterol analyses, which were done by the gas chromatographic method (30). The technical assistance of Mrs. Sarah Massey is gratefully acknowledged.

This work was supported by grants HL19168 and Program Project No. HL22633, National Heart, Lung, and Blood Institute, National Institutes of Health, Bethesda, Md.

\section{REFERENCES}

1. Marsh, J. B., and D. L. Drabkin. 1960. Experimental reconstruction of metabolic pattern of lipid nephrosis: key role of hepatic protein synthesis. Metab. Clin. Exp. 9: 946-955.

2. Radding, C. M., and D. Steinberg. 1960. Studies on the synthesis and secretion of serum lipoproteins by rat liver slices. J. Clin. Invest. 39: 1560-1568.

3. Marsh, J. B. 1974. Lipoproteins in a nonrecirculating perfusate of rat liver. J. Lipid Res. 15: 544-550.

4. Gherardi, E., E. Rota, S. Calandra, R. Genova, and A. Tamborino. 1977. Relationship among the concentrations of serum lipoproteins and changes in their chemical composition in patients with untreated nephrotic syndrome. Eur. J. Clin. Invest. 7: 563-570.

5. Rudel, L. L., J. A. Lee, M. D. Morris, and J. M. Felts. 1974. Characterization of plasma lipoproteins separated and purified by agarose column chromatography. Biochem. J. 139: 89-95.

6. Lux, S. E., K. M. John, and H. B. Brewer. 1972. Isolation and characterization of apoLp-Gln-11 (Apo A-11), a plasma high density lipoprotein containing two identical polypeptide chains. J. Biol. Chem. 247: 7510-7518.

7. Marsh, J. B., and D. B. Weinstein. 1966. Simple charring method for determination of lipids. J. Lipid Res. 7: 574576.

8. Zlatikis, A., B. Zak, and A. J. Boyl. 1953. A new method for the direct determination of serum cholesterol. J. Lab. Clin. Med. 41: 486-492.

9. Marsh, J. B. 1976. Apoproteins of the lipoproteins in a non-recirculating perfusate of rat liver. J. Lipid Res. 17: 85-90.

10. Kane, J. P., T. Sata, R. L. Hamilton, and R. J. Havel. 1975. Apoprotein composition of very low density lipoproteins of human serum. J. Clin. Invest. 56: 1622-1634.

11. Swaney, J. B., H. Reese, and H. A. Eder. 1974. Polypeptide composition of rat high density lipoprotein: characterization by SDS-Gel electrophoresis. Biochem. Biophys. Res. Commun. 59: 513-519.

12. Lowry, O. H., N. J. Rosebrough, A. L. Farr, and R. J. Randall. 1951. Protein measurement with the Folin phenol reagent. J. Biol. Chem. 193: 265-275.

13. Marsh, J. B. 1978. Labeling of high density lipoproteins with $\left[{ }^{3} \mathrm{H}\right]$ acetic anhydride. J. Lipid Res. 19: 107-110.

14. Shafrir, E., T. Brenner, A. Gutman, and M. Orevi. 1974. Hepatic metabolic pattern in experimental nephrotic syndrome: glycolysis, gluconeogenesis, and amino acid metabolism. Am. J. Physiol. 226: 162-167.

15. Nakaya, N., B. H. Chung, and O. D. Taunton. 1977. Synthesis of plasma lipoproteins by the isolated perfused liver from the fasted and fed pig. J. Biol. Chem. 252: 5258-5261.

16. Marsh, J. B., and D. L. Drabkin. 1958. Metabolic channeling in experimental nephrosis III influence of diet and of adrenalectomy; liver hypertrophy. J. Biol. Chem. 230: 1063-1071.

17. Marsh, J. B., and C. E. Sparks. 1979. Lipoproteins in experimental nephrosis: plasma levels and composition. Metab. Clin. Exp. In press.

18. Marsh, J. B., and D. L. Drabkin. 1955. Metabolic channeling in experimental nephrosis II lipid metabolism. J. Biol. chem. 212: 633-639.

19. Hamilton, R. L., M. C. Williams, C. J. Fielding, and R. J. Havel. 1976. Discoidal bilayer structure of nascent high density lipoproteins from perfused rat liver. J. Clin. Invest. 58: $667-680$.

20. Fainaru, M., T. E. Felker, R. L. Hamilton, and R. J. Havel. 
1977. Evidence that a separate particle containing $\beta$-apoprotein is present in high density lipoproteins from perfused rat liver. Metab. Clin. Exp. 26: 999-1004.

21. Drabkin, D. L., J. B. Marsh, and G. A. Braun. 1962. Amino acid mobilization in plasma protein biosynthesis in experimental nephrosis. Metab. Clin. Exp. 11: 967-977.

22. Mayer, M., and E. Shafrir. 1972. Liver and plasma amino acid pattern in glucogenic conditions and in nephrotic syndrome. Israel J. Med. Sci. 8: 859-861.

23. Peters, T. 1953. Evidence of intermediate compounds in serum albumin synthesis. J. Biol. Chem. 200: 461-470.

24. Fainaru, M., M. C. Glangeaud, and S. Eisenberg. 1975. Radioimmunoassay of human high density lipoprotein apoprotein A-1. Biochim. Biophys. Acta. 386: 432-443.

25. Felker, T. E., M. Fainaru, R. L. Hamilton, and R. J. Havel. 1977. Secretion of the arginine-rich and A-1 apolipoproteins by the isolated perfused rat liver. J. Lipid Res. 18: 465-473.
26. Eisenberg, S., D. W. Bilheimer, and R. J. Levy. 1972. The metabolism of very low density lipoprotein proteins 11: Studies on the transfer of apoproteins between plasma lipoproteins. Biochim. Biophys. Acta. 280: 94-104.

27. Derr, R. F., D. K. Loechler, C. S. Alexander, and H. T. Nagasaw. 1968. Inhibition of aminonucleoside nephrosis in rats. (IV) Prevention by $\mathrm{N}^{6}$-Methyladenosine. J. Lab. Clin. Med. 72: 363-369.

28. Sparks, C. E., and J. B. Marsh. 1978. Very low density lipoprotein catabolism in experimental nephrosis. Fed. Proc. 37: 1321. (Abstr.)

29. Staprans, I., and J. M. Felts. 1977. The effect of $\alpha$-acid glycoprotein (orosomucoid) on triglyceride metabolism in the nephrotic syndrome. Biochem. Biophys. Res. Commun. 79: $1272-1278$.

30. Bates, S. R., and G. H. Rothblat. 1974. Regulation of cellular sterol flux and synthesis by human serum lipoproteins. Biochim. Biophys. Acta. 360: 38-55. 